\title{
FACTORES DE RIESGO PARA LA RECURRENCIA DE HERNIA DE DISCO LUMBAR
}

\author{
FATORES DE RISCO DE RECORRÊNCIA DE HÉRNIA DE DISCO LOMBAR
}

RISK FACTORS FOR RECURRENT LUMBAR DISC HERNIATION

Angel Rodríguez Segura ${ }^{1}$, Enrique Medellín López², Roberto Chapa Sosa ${ }^{3}$

\section{RESUMEN}

Objetivo: La recurrencia de hernia del disco lumbar es un padecimiento que implica algunas dificultades en relación con el diagnóstico y tratamiento. Es importante tratar de identificar los factores que puedan influir en la presentación de la recurrencia. Métodos: Se revisaron los casos de pacientes operados por hernia de disco lumbar recurrente entre los años de 2006 y 2008, buscando identificar los factores que tuvieran relación con dicha patología, y también se revisaron los casos de pacientes operados por hernia de disco lumbar en forma primaria. Resultados: Se analizaron los casos de 20 pacientes con hernia discal lumbar recurrente y de 27 con hernia discal lumbar operados en forma primaria. Para los pacientes con recurrencia, se encontró que el 95\% de ellos presentaban algún grado de obesidad, determinado por la Fórmula de Quetelet basada en el Índice de Masa Corporal; se observó también que el 75\% de los casos tenían protrusión, con resultados excelentes en $35 \%$, y buenos en $55 \%$, el $10 \%$ presentó molestias residuales de forma grave. Para los pacientes con hernia primaria se estudiaron 27 casos, en los que se observó que el $92 \%$ de ellos se operaron antes de los 6 meses de iniciado el cuadro, 38\% tuvieron resultados excelentes, 40\% buenos y el 19\% malos resultados. Al estudiar las variables, con el índice de Pearson, se encontró relación de la recurrencia con tabaquismo, trabajo, diabetes e hipertensión, y nivel de estudios, no con la obesidad. Conclusiones: Es destacable que dos de los factores relacionados son susceptibles de modificación antes del evento quirúrgico o posteriormente a este, lo cual puede beneficiar el desenlace del mismo.

Descriptores: Recurrencia; Desplazamiento del disco intervertebral; Columna vertebral/cirugía; Procedimientos ortopédicos/efectos adversos; Estudios retrospectivos.

\section{RESUMO}

Objetivo: A hérnia de disco envolve algumas dificuldades com relação ao diagnóstico e tratamento. É importante identificar os fatores que podem influenciar a recorrência. Métodos: Foram analisados casos de pacientes operados de hérnia de disco lombar recidivante entre 2006 e 2008 , buscando identificar os fatores que têm relação com a doença; também se analisaram os casos de pacientes submetidos à cirurgia primária de hérnia de disco lombar. Resultados: Foram analisados os casos de 20 pacientes com hérnia de disco lombar recidivante e 27 casos de cirurgia primária de hérnia de disco lombar. Para os pacientes com recorrência, constatou-se que 95\% tinham algum grau de obesidade, determinada pela fórmula de Quetelet, baseada no Índice de Massa Corporal; e também foi obsenvado que 75\% dos casos eram protrusão do disco com excelentes resultados em 35\% e bons em 55\%; 10\% tiveram problemas residual grave. Foram estudados 27 casos de pacientes com hérnia primária, nos quais se verificou que 92\% foram operados antes dos 6 meses do início do quadro, 38\% tiveram resultados excelentes, 40\%, bom e 19\%, resultado ruim. Ao considerar as variáveis com a relação de Pearson encontrou-se relação da recorrência com tabagismo, trabalho, diabetes, hipertensão e escolaridade, mas não com a obesidade. Conclusão: É importante ressaltar que dois dos fatores estão sujeitos a alteração antes da cirurgia ou depois dela, o que pode beneficiar o resultado do mesmo.

Descritores: Recidiva; Deslocamento do disco intervertebral, Coluna vertebral/cirurgia; Procedimentos ortopédicos/efeitos adversos; Estudos retrospectivos.

\begin{abstract}
Objective: Recurrent lumbar disc herniation is a condition that involves some difficulties in relation to diagnosis and treatment. It is important to identify factors that may influence recurrence. Methods: We reviewed cases of patients operated for recurrent lumbar disc herniation between 2006 and 2008 , seeking to identify the factors that have relation with this disease, and also reviewed the cases of patients that underwent primary surgery for herniated lumbar disc. Results: We analyzed the cases of 20 patients with recurrent lumbar disc herniation and 27. For patients with recurrence, it was found that $95 \%$ had some degree of obesity determined by the Quetelet Index, based on Body Mass Index; it was also observed that $75 \%$ of cases were protruding with excellent results in 35\% and good in 55\%, 10\% had severe residual conditions. In patients with primary hernia were studied 27 cases in which it was noted that $92 \%$ were operated before 6 months of the onset of the condition, 38\% had excellent results, $40 \%$ good and $19 \%$ poor outcomes. Considering the variables with the Pearson correlation, it was found that the recurrence is related to smoking, work, diabetes and hypertension, and schooling, but not with obesity. Conclusions: It is remarkable that two of the factors are subject to change before or after the surgery, which can benefit the ir outcomes.
\end{abstract}

Keywords: Recurrence; Intervertebral disk displacement; Spine, Surgery; Orthopedic procedures, Adverse Effects; Retrospective studies.

\section{INTRODUCCIÓN}

La lumbalgia ha afligido al ser humano por muchos miles de años. Hay descripciones de lumbalgia y ciática en la Biblia y en los escritos de Hipócrates. Apesar de la larga historia de este padeci- miento, la explicación razonable y científica del origen de la lumbalgia y del dolor en la pierna surgió hasta 1934, con la publicación del escrito clásico de Mixter y Barr ${ }^{1}$. La enfermedad humana asume importancia en términos de muerte y de incapacidad, y la enfermedad de la

1. Médico Cirujano Ortopedista, Servicio de Ortopedia y Traumatología, Hospital General de Zona No. 1, Instituto Mexicano del Seguro Social - Zacatecas, México.

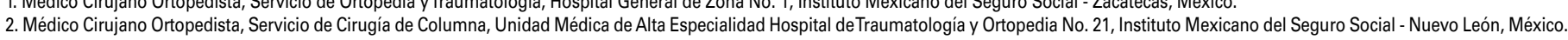

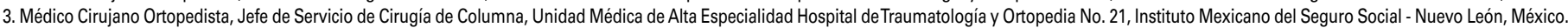

Trabajo realizado en Unidad Médica de Alta Especialidad, Hospital deTraumatología y Ortopedia No. 21, Instituto Mexicano del Seguro Social, Monterrey, Nuevo León, México. Correspondencia: Privada Sierra Mojada \# 205, Fraccionamiento Lomas de San Francisco, Código Postal 98067, Zacatecas, México. E-mail: yoman59@ hotmail.com 
columna vertebral aunque si bien no es letal, si tiene importancia en cuanto al impacto en la población en relación a dolor e incapacidad².

Nachemson ${ }^{3}$ estimó que en algún momento de la vida adulta, el $80 \%$ de las personas experimentará lumbalgia en grado importante, además mostró que solo el $35 \%$ de estos pacientes desarrollaron ciática, aunque se estima que solo entre el $4 \%$ y el $6 \%$ de las hernias discales son sintomáticas ${ }^{4}$, y que una vez que desaparece el ataque original de lumbalgia, hasta el 90\% tuvieron recurrencia del dolor ${ }^{2}$.

Los datos de prevalencia indican que $4.8 \%$ de los varones y $2.5 \%$ de las mujeres mayores de 35 años experimentarán ciática. Se ha informado ${ }^{2}$ que $75 \%$ de estos pacientes mejoraban 10 a 30 días después del inicio de los síntomas y que solo el 19\% requería de tratamiento quirúrgico tarde o temprano.

Dentro de la evolución natural de la hernia de disco lumbar se encontró que al año de evolución, el tratamiento quirúrgico resulto mejor que el tratamiento no operatorio en términos de alivio del dolor y recuperación funcional ${ }^{5}$, sin embargo, después de 4 años, el gru-

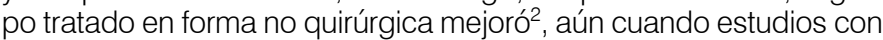
seguimiento a 25 años y más reportan calidad de vida satisfactoria y mayor alivio del dolor que los pacientes no operados 6 . Por lo que es razonable la preferencia de los pacientes a aceptar el manejo quirúrgico una vez explicadas las alternativas ${ }^{7}$

Estudios similares han mostrado que el tratamiento no quirúrgico muestra morbilidad implícita más prolongada, mejoría lenta pero definitiva y resultados aceptables aunque tal vez no ideales ${ }^{2,4}$.

Los resultados de cirugía primaria para hernia de disco intervertebral lumbar son reportados como muy buenos por la mayoría de los autores, con retorno a las actividades laborales con una capacidad similar al preoperatorio hasta en un $84 \%^{8}$, y en diversos estudios se ha mostrado que entre los factores principales para la recuperación se encuentra el tipo de hernia, el nivel, la historia de dolor ciático predominante sobre el lumbar, el tabaquismo, la limitación laboral y la compensación laboral previa al evento quirúrgico 8-10.

Los reportes de recurrencia difieren poco en la literatura, y siempre en relación al tiempo, hasta una tasa de $7.1 \%$ a los 8.5 años ${ }^{11}$, y posterior a este tiempo sin recurrencias demostradas. Uno de los factores atribuidos a la recurrencia es la cantidad de disco que es removida, por lo que McGirt et al. ${ }^{12}$ publican un estudio en 2009, donde reportan los resultados de la revisión de la literatura que comprende a 13,359 pacientes divididos en 2 grupos de acuerdo a la cantidad de disco retirado y observan que la tasa de recurrencia varía de 7\% (con la discectomía limitada, popularizada por Williams ${ }^{13}$ y Sprengler ${ }^{14}$, que consta en la remoción del fragmento herniado, con poca invasión al espacio discal) hasta 3.5\% (con la discectomía agresiva, popularizada por $\mathrm{O}{ } \mathrm{C}^{\mathrm{C}}$. remoción del fragmento herniado más curetaje del disco remanente.

En cuanto a los resultados de la cirugía de revisión por hernia discal lumbar, Papadopoulos et al. ${ }^{16}$ realizan un estudio comparando sus resultados con los obtenidos en cirugías primarias y no encuentra diferencias significativas entre ambos, por lo que concluye que la cirugía de revisión es eficaz para tratar el dolor.

Existen diversos estudios acerca de la importancia de la correcta interpretación de los estudios de imagen, tanto en pacientes con hernia primaria o recurrente ${ }^{17-19}$

La correcta interpretación de estos estudios de imagen asociados a los datos clínicos nos permite la selección de manejo más adecuado a cada uno de los casos. Aún permanece controversial el uso de técnicas diagnósticas como el caso de la discografía ${ }^{20}$.

\section{MATERIAL Y MÉTODOS}

\section{Clasificación del diseño del estudio:}

Sin intervención, observacional, analítico, correlacional, transversal, retrospectivo.

\section{Población de estudio}

Pacientes sometidos a tratamiento quirúrgico por diagnóstico de hernia de disco lumbar en un solo nivel, en forma primaria, en la UMAE No. 21, IMSS, Monterrey, N. L., México (Figura 1A y B, Caso 1).

\section{Criterios de Grupo 1, hernias recurrentes:}

a) Criterios de inclusión:

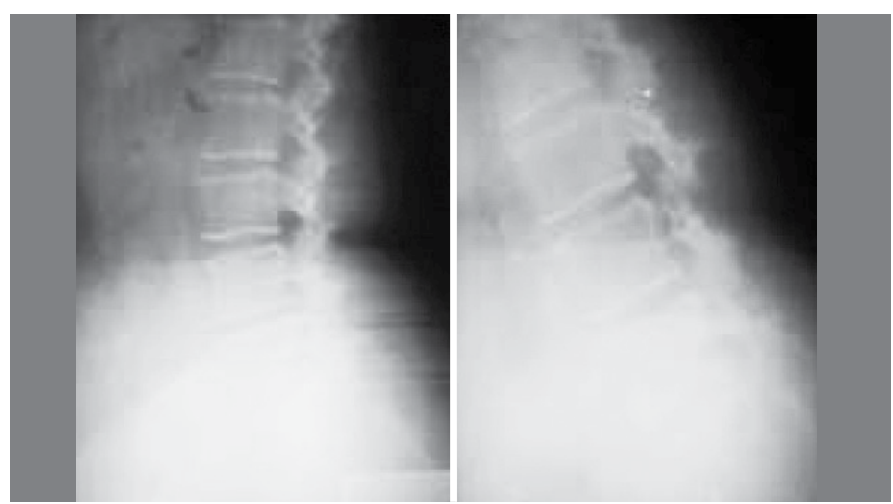

Figura 1A . Caso 1. Radiografías preoperatorias de paciente femenino de 58 años de edad, quien 2 años antes se había intervenido por hernia de disco L4 - L5.
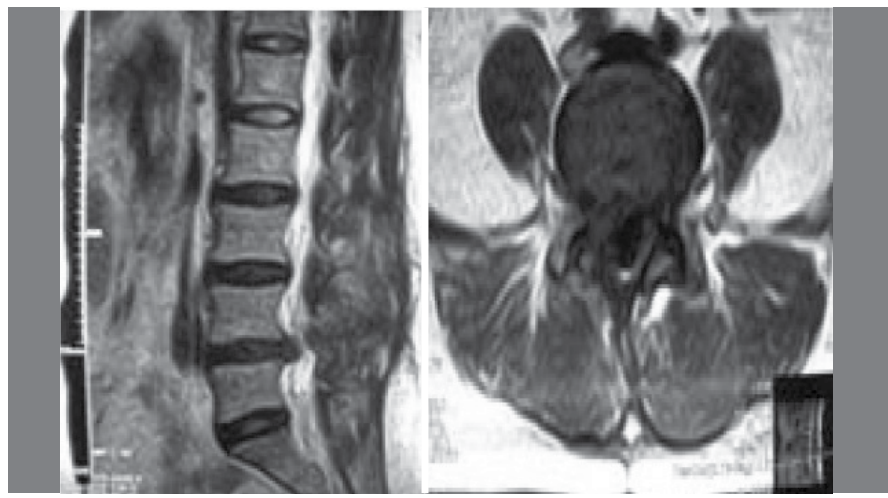

Figura 1B. Caso 1. Resonancia magnética de paciente femenino de 58 años de edad, donde se observa la recurrencia de la hernia discal L4 - L5 derecha.

a. Pacientes con hernia de disco lumbar a un solo nivel, con diagnóstico clínico y de gabinete coincidente;

b. Pacientes que hayan recibido tratamiento quirúrgico consistente en excisión simple del disco lumbar herniado;

c. Pacientes que posterior a la cirugía tuvieron mejoría de los síntomas;

d. Pacientes que tras un alivio temporal presenten diagnóstico de hernia de disco recurrente, tanto clínico como de gabinete.

b) Criterios de exclusión:

a. Pacientes con patología de columna concomitante a la patología discal, como inestabilidad, estenosis, infecciones, tumores o fracturas;

b. Pacientes que no hayan reportado alivio del dolor tras la cirugía

c. Pacientes en los cuales el diagnóstico clínico y el radiológico no sea coincidente y concluyente;

c) Criterios de eliminación:

a. Expediente clínico incompleto;

Criterios de Grupo 2, hernias primarias (Figuras 2 A, B, C y D, Caso 2):

a) Criterios de inclusión:

a. Pacientes con hernia de disco lumbar a un solo nivel, con diagnóstico clínico y de gabinete coincidente;

b. Pacientes que hayan recibido tratamiento quirúrgico consistente en excisión simple del disco lumbar herniado;

c. Pacientes que posterior a la cirugía tuvieron mejoría de los síntomas

b) Criterios de exclusión:

a. Pacientes con patología de columna concomitante a la patología discal, como inestabilidad, estenosis, infecciones, tumores o fracturas;

b. Pacientes que no hayan reportado alivio del dolor tras la cirugía;

c. Pacientes en los cuales el diagnóstico clínico y el radiológico no sea coincidente y concluyente.

c) Criterios de eliminación:

a. Expediente clínico incompleto;

b. Pacientes que durante el estudio desarrollen recurrencia de la hernia de disco. 


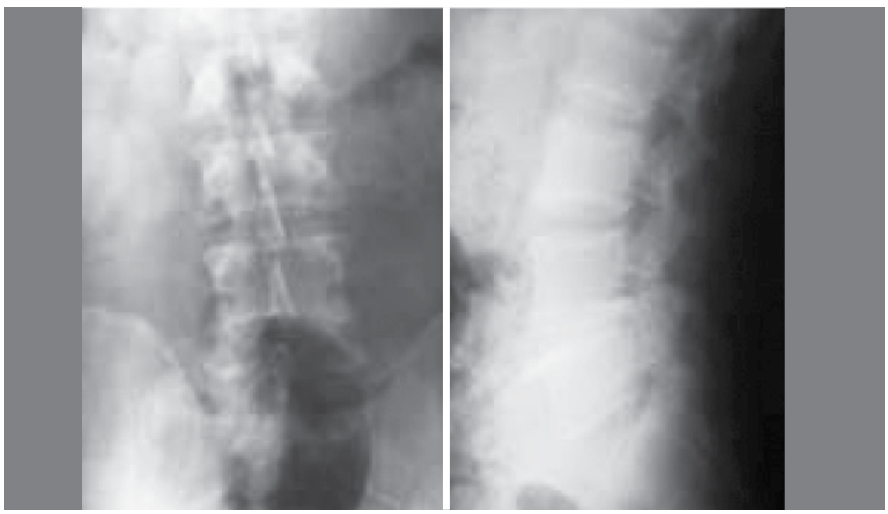

Figura 2A. Caso 2. Masculino de 38 años, cirugía por hernia de disco lumbar L4 - L5 izquierda, radiografías preoperatorias.
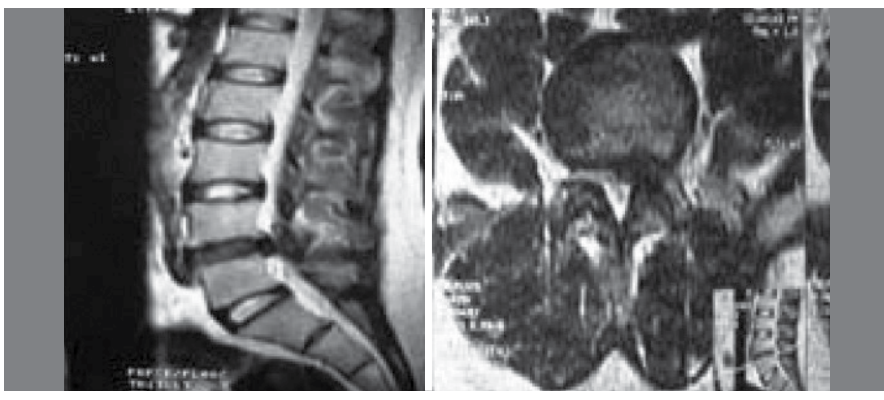

Figura 2B. Caso 2. Resonancia magnética preoperatoria, masculino de 38 años.

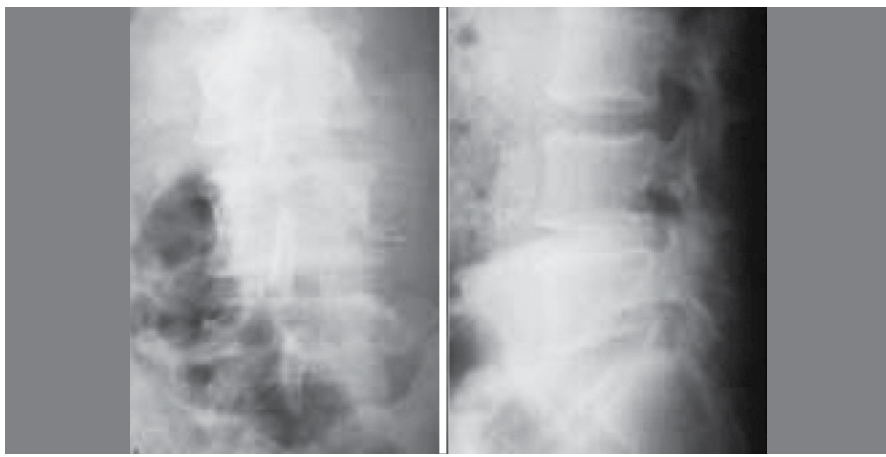

Figura 2C. Caso 2. Radiografías preoperatorias a la cirugía de revisión, masculino de 38 años.

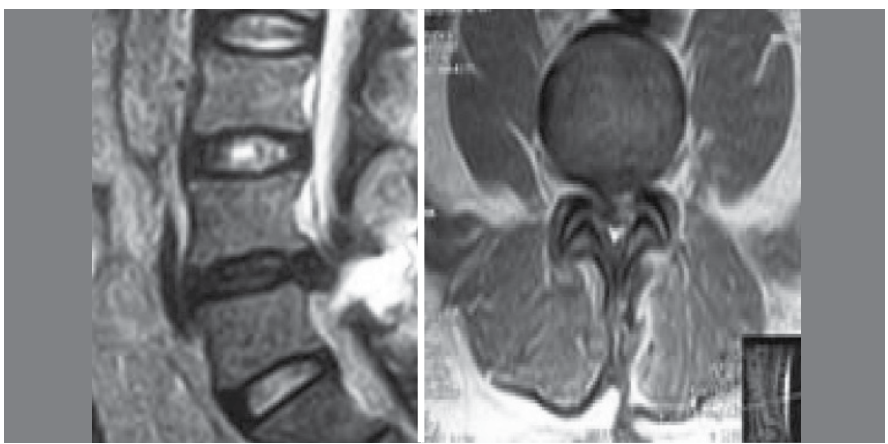

Figura 2D. Caso 2. Resonancia magnética previa a la cirugía de revisión, masculino de 38 años.

\section{MUESTRA}

Población derechohabiente al IMSS, sometida a tratamiento quirúrgico por hernia discal lumbar a un solo nivel, durante el periodo comprendido desde el 10. de enero 2006 hasta el 31 de diciembre del 2008, que incluyera criterios de inclusión descritos (Figura $3 \mathrm{~A}$ y $\mathrm{B}$ - Caso 3).
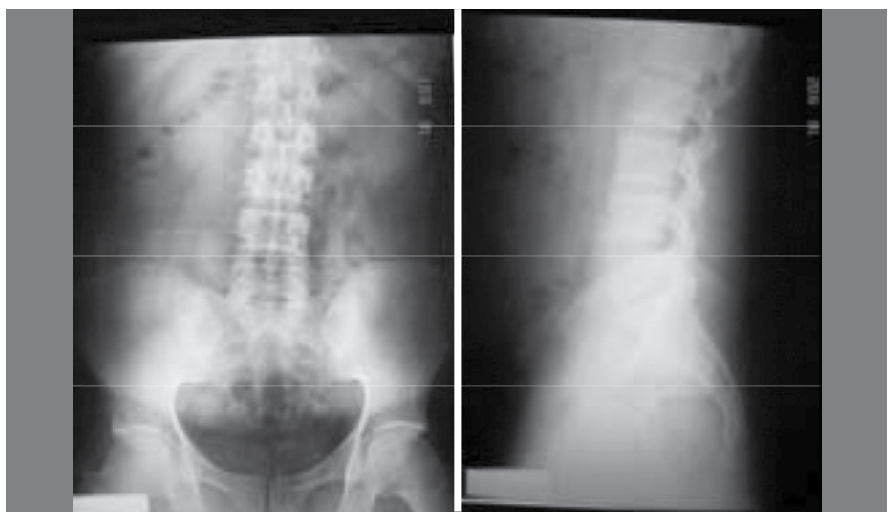

Figura 3A. Caso 3. Radiografías preoperatorias. Masculino de 32 años. Con hernia de disco L5 - S1 derecha.

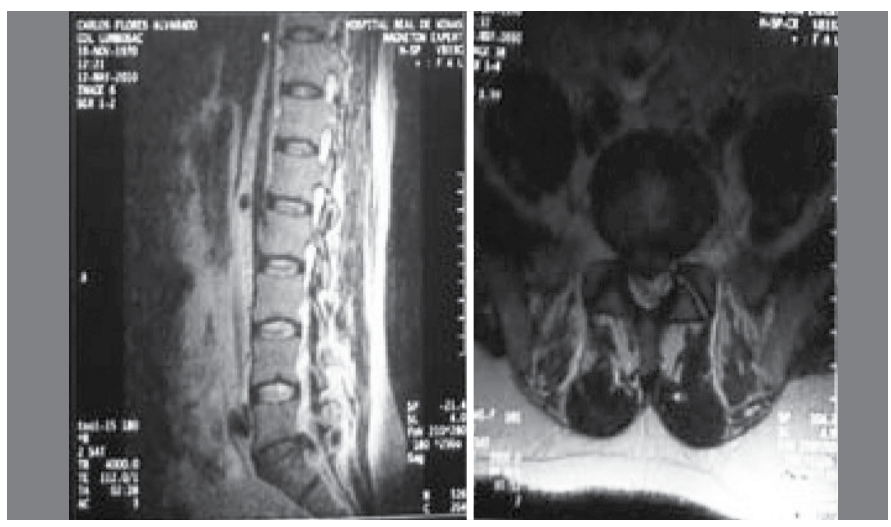

Figura 3B. Caso 3. Resonancia magnética que muestra la hernia de disco L5 - S1 derecha.

\section{TÉCNICA QUIRÚRGICA}

Los pacientes son operados en posición supina, con colocación de rollos de soporte torácico para permitir la ventilación adecuada y la descompresión del abdomen, con protección de puntos de apoyo y rostro. Se aplica una dosis de antibiótico profiláctico una hora previa al evento quirúrgico. Se realiza la técnica estándar para laminectomía y discectomía abierta con el apoyo de radiografía transoperatoria para confirmar nivel. El tiempo habitual del procedimiento es de 40 minutos, con sangrado mínimo. En los casos en que se encuentran cambios inflamatorios macroscópicos en la raíz se aplica localmente una dosis de 80 mgrs de acetato de metilprednisolona. Habitualmente se coloca gelfoam sobre el espacio de laminectomía. Se permite la movilización en cama desde el postoperatorio inmediato y a las 12 horas la deambulación, con egreso hospitalario a las 48 horas. El control subsecuente es a las 2, 6 y 10 semanas del postoperatorio.

\section{PLAN DE ANÁLISIS}

Se analizaron los resultados mediante frecuencias y correlación de Pearson (R de Pearson). Se utilizó el software del SPSS versión 10 (Figuras 4 a 12).

Grupo 1: Hernias recurrentes, Grupo 2: Grupo control, hernias primarias.

\section{RESULTADOS}

\section{Grupo 1:}

Se encontró un total de 20 pacientes para ser incluidos en el estudio, cumpliendo todos los criterios de inclusión y depurando aquellos con criterios de exclusión o eliminación, de los cuales se obtuvo información para efectuar análisis de frecuencias y de correlación de Pearson. Los pacientes con sobrepeso u obesidad sumaron el $95 \%$ de los casos. Las protrusiones tuvieron el $75 \%$ de 


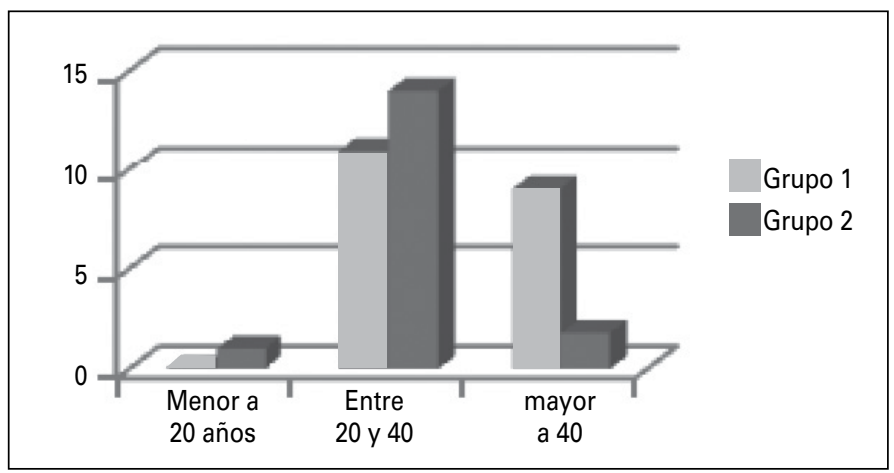

Figura 4. Distribución por sexo.

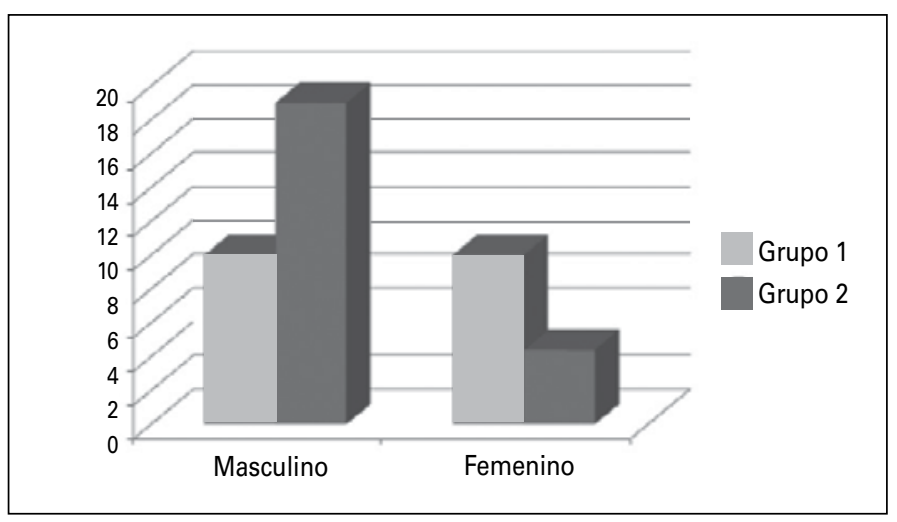

Figura 5. Distribución por edades.

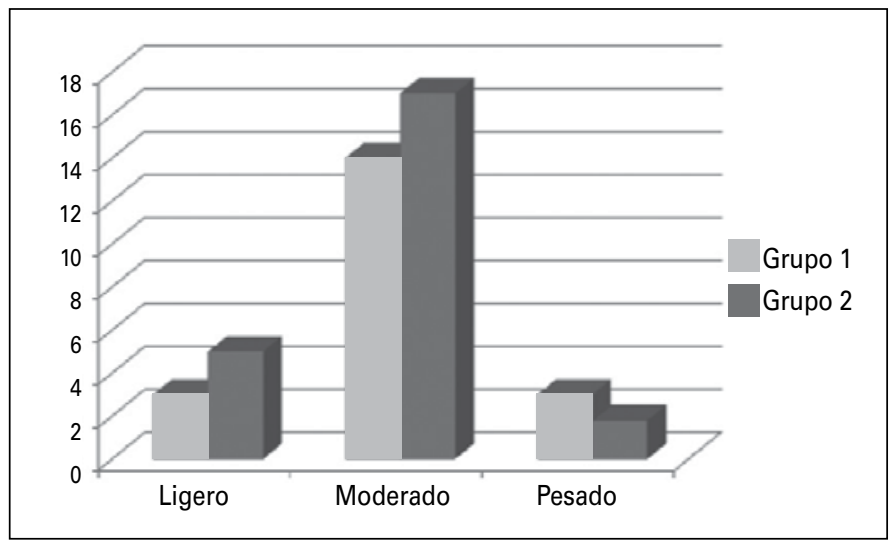

Figura 6. Distribución por tipo de trabajo.

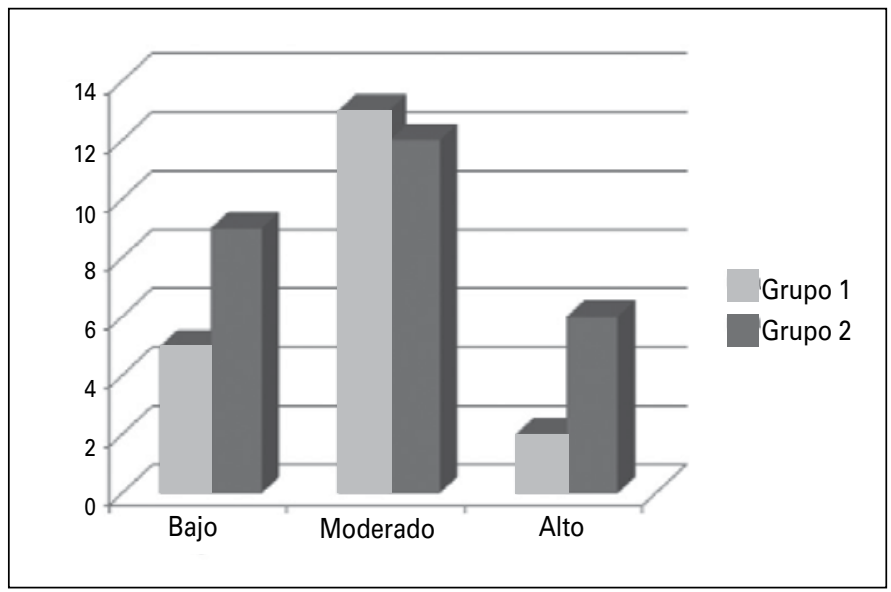

Figura 7. Distribución por nivel de escolaridad. frecuencia. El 35\% tuvo excelente resultado final, sin dolor lumbar o radicular residual, reintegrándose a su actividad habitual; el 55\% de los pacientes tuvo lumbalgia residual, pero sin mayor limitación para las actividades de su vida diaria, solo el 10\% persistió con molestias en forma severa, requiriendo modificaciones importantes en sus actividades habituales. Para los pacientes reoperados, la tasa de lumbalgia residual es alta (85\%), pero sin casos de radiculopatía residual.

\section{Grupo 2:}

Se obtuvo información de 40 pacientes seleccionados en forma aleatoria, usando como criterio de selección la fecha de cirugía que coincidiera con la de los pacientes del grupo 1, o en su defecto la fecha más próxima a esta.

De estos 40 pacientes se eliminaron 13 pacientes por los criterios de eliminación o exclusión. Con esto, tuvimos un total de 27 pacientes para obtener información para efectuar los análisis de frecuencias y de correlación de Pearson.

El 92\% de los pacientes se opera antes de los 6 meses de evolución, con más de $60 \%$ de protrusiones. El 38\% de los pacientes manifiestan un resultado excelente, $40 \%$ satisfactorio, sin cambios significativos en sus actividades habituales, y en el 19\% de los pacientes el resultado es malo, con ciática residual o por lo menos la incapacidad de realizar sus actividades habituales en forma normal, mientras que el resto de las variables estudiadas no mostraron diferencias de interés (Figura 8).

Al aplicar el análisis de $\mathrm{R}$ de Pearson a la variable recurrencia, se encontró lo siguiente: (Tabla 1 y Figuras 9 y 10).

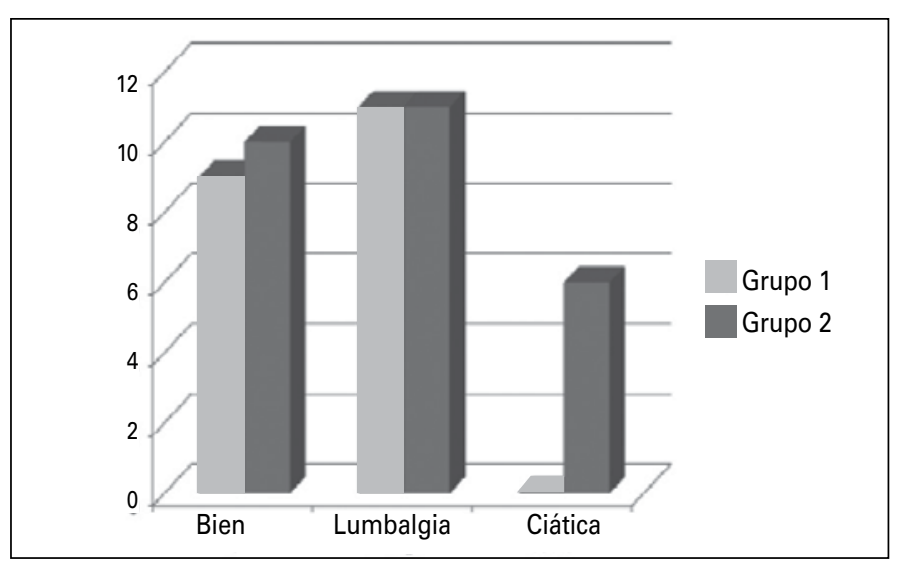

Figura 8. Distribución por resultado final.

Tabla 1. Correlación entre recurrencia y factor estudiado.

\begin{tabular}{c|c}
\hline Variables & Recurrencia \\
\hline Edad & .346 \\
\hline Sexo & .084 \\
\hline Trabajo & .500 \\
\hline Escolaridad & .400 \\
\hline Tabaquismo & .647 \\
\hline Peso & .004 \\
\hline Diabetes & .480 \\
\hline Hipertensión & .369 \\
\hline Otras enfermedades & .034 \\
\hline Tipo de hernia & .190 \\
\hline Nivel de la lesión & .317 \\
\hline Lado de lesión & .328 \\
\hline Evolución & .319 \\
\hline Resultado & .190 \\
\hline Incapacidad & .153 \\
\hline Alta del servicio & .347 \\
\hline Alta laboral & .275 \\
\hline
\end{tabular}




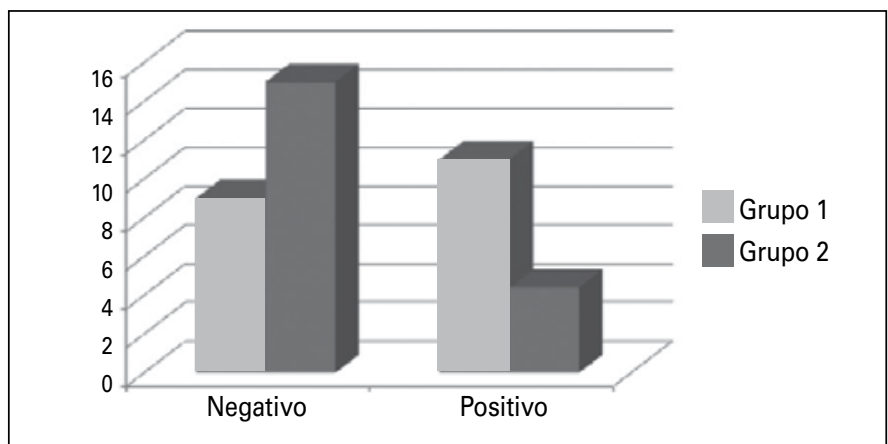

Figura 9. Distribución por tabaquismo.

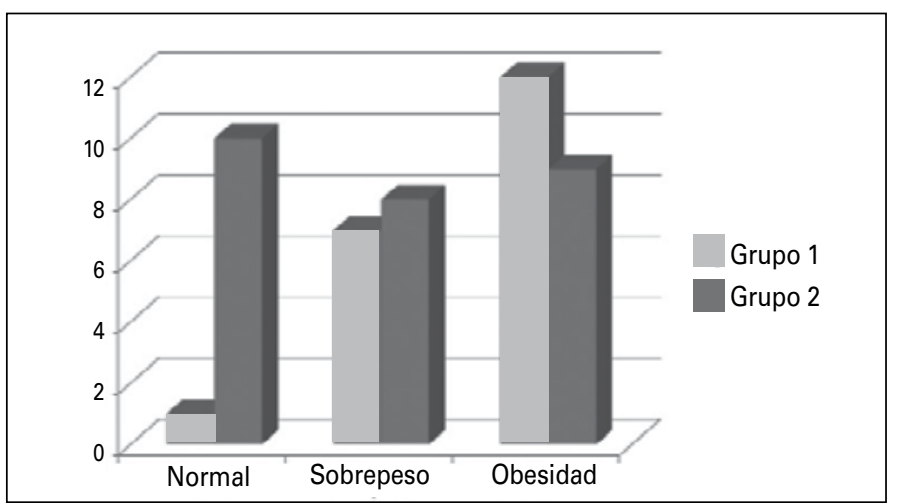

Figura 10. Distribución por peso.

\section{DISCUSION}

El objetivo general de la investigación fue el de determinar los factores que influían en la presentación de recurrencia de hernia discal, y entre las variables estudiadas se encontraron algunas de ellas no reportadas en la literatura como el tabaquismo, la diabetes y la hipertensión arterial, el nivel de escolaridad, etc. tenían relación significativa con la recidiva, mientras que factores de riesgo encontrados en la literatura tales como el sexo, el peso y el tipo de hernia no se encontraron de importancia en el presente estudio ${ }^{21}$ (Tabla 1).

En el estudio a largo plazo de Loupasis, hay un alto porcentaje de resultados insatisfactorios (36\%), tanto por dolor lumbar residual, recurrencia de los síntomas radiculares o ambos. En su estudio, el sexo femenino fue predictivo de malos resultados, mientras que los factores laborales tuvieron influencia en la prevalencia de síntomas residuales. También encontraron que el nivel educativo se relaciono en forma positiva con el resultado final ${ }^{22}$. En nuestro estudio no observamos esta tendencia con respecto al sexo femenino, aunque si hubo relación con el tipo de actividad laboral.

Aunque, por otro lado, en estudios también recientes se muestra que los hallazgos transoperatorios como son el tipo de hernia, el tamaño del defecto anular y los hallazgos radiológicos preoperatorios como es el tamaño discal se relacionan mas a la recurrencia que los datos demográficos antes mencionados ${ }^{23}$.

En el estudio de Carragee et al. ${ }^{24}$ del 2003, los pacientes con fragmentos extruidos y mínimo daño al anillo fibroso tuvieron mejor resultado que aquellos con fragmentos contenidos (extruidos) y defectos grandes del anillo, por su alto índice de recurrencia y reintervenciones, lo cual reviste importancia para nuestras observaciones, ya que nos indica la importancia de señalar claramente las características de la lesión observadas transoperatoriamente. Lo mismo podemos mencionar acerca del volumen retirado de disco, ya que esto tiene que ver con la recurrencia y con la pérdida de altura del disco. El peso no afecta a la recurrencia, pero si a la perdida de altura del disco 25 .

Yorimitsu et al. ${ }^{26}$ publican su trabajo de revisión de resultados a largo plazo en el que encontró que el dolor lumbar residual se presenta en la mayoría de los casos, aunque de intensidad discreta. En los pacientes con dolor severo, observo que tenían 35 años o menos al momento de la cirugía y que mostraron degeneración discal importante, por lo que en sus conclusiones recomienda la fusión primaria. Por otro lado, los pacientes que mantenían la altura discal, evolucionaban con menos dolor lumbar, pero con un mayor índice de recurrencia. En nuestros casos, no recomendamos la fusión primaria a menos que se encuentre algún tipo de inestabilidad asociada a la hernia discal.

Una de las dificultades para el diagnóstico de hernia recurrente es la presencia de fibrosis postoperatoria, la cual puede ser la causa de molestias residuales o recidivantes hasta en un 5\% de los casos. El lasegue, la incapacidad para deambular y dolor al toser son más característicos de hernia discal, en cambio el uso de analgésicos está más asociado a la fibrosis ${ }^{27}$.

Se demostró que la tasa de recurrencia aumenta con el paso del tiempo, Gaston y Marshall encontraron en su estudio publicado en el 2003 que se incrementaba desde 1.1\% a un año, hasta 5.0\% a 5 años y $7.9 \%$ a 8 años ${ }^{28}$.

La incidencia de cirugía por hernia discal recurrente en el hospital se encontró en $5.4 \%$ para los 3 años estudiados y constituyeron el 2.3\% de la programación de cirugías, lo cual se encuentra en los parámetros más bajos reportados en la literatura.

Es interesante señalar que el diagnóstico de recurrencia de hernia discal no tuvo diferencia significativa en relación al tiempo transcurrido desde la presentación de los síntomas o estudios realizados comparándolo con las hernias primarias, lo cual habla de una eficaz interpretación de los datos clínicos y los hallazgos radiológicos tal y como se señala en estudios recientes publicados. En estos se menciona la presentación clínica de la recurrencia contra la sintomatología debida a la fibrosis peridural, así como su interpretación radiológica. En este último caso, es de resaltar la importancia de los métodos más actuales de clasificación de los hallazgos para la estandarización de los diagnósticos, como en la clasificación de la Universidad Estatal de Michigan ${ }^{29}$. Es muy importante realizar el diagnóstico diferencial para ofrecer la mejor opción terapéutica a los pacientes, pero también tiene su aspecto económico, ya que el costo del manejo quirúrgico de una recurrencia se dispara comparado con el costo del manejo quirúrgico de una discectomía primaria ${ }^{30}$.

Los pacientes que se someten a cirugía por hernia discal recurrente tuvieron resultados equiparables a los pacientes con hernia primaria, no presentando posterior al evento ciática residual, aunque si lumbalgia, sin ser factor de limitación física.

Esto está de acuerdo a lo reportado en la literatura y lleva al planteamiento de la necesidad de una fusión en los casos de reintervención, sin embargo en nuestro hospital no se realiza en forma rutinaria, aun cuando se encuentre hernia contralateral, a excepción de los casos en que está demostrada una inestabilidad por cualquier causa, lo cual también basado en lo demostrado por diferentes estudios ${ }^{31-33}$.

Los resultados funcionales y la tasa de satisfacción de los pacientes reoperados fueron similares a lo encontrado en los pacientes con discectomía primaria, por lo que no se recomienda la realización de procedimientos adicionales a la discectomía contralateral, a pesar de que biomecánicamente se altera el segmento involucrado ${ }^{32}$.

A largo plazo, el dolor lumbar residual es causa de mal resultado, lo que puede deberse a daño muscular por exposición prolongada como en el caso de la fusión. Por esto no realizamos la fusión de primera intención ${ }^{33}$.

De los factores observados, los dos con mayor relación a la recurrencia son el tabaquismo y la actividad laboral, que son factores susceptibles de modificación. Los demás factores, no están en condiciones de modificarse, por lo que solo queda el hacer el análisis de ellos que permita la toma de decisiones y el informar al paciente de los probables riesgos y beneficios del manejo quirúrgico para hernia de disco. (Figuras 11 y 12).

Los pacientes se sienten más satisfechos con el tratamiento antes y después de la cirugía que con la información preoperatoria dada. Esto, nos indica claramente que se requiere tomar un tiempo suficiente para explicar y asegurarnos de la comprensión de los objetivos, beneficios, riesgos y posibles complicaciones de los tratamientos. Además, se mostró que los pacientes con expectativas claras y reales en relación al retorno laboral y a la recuperación del estado de salud se muestran más satisfechos con los resultados de la cirugía ${ }^{34}$.

En nuestro medio, no está extendido el uso de materiales o técnicas para reparar el defecto causado por la extracción de disco 


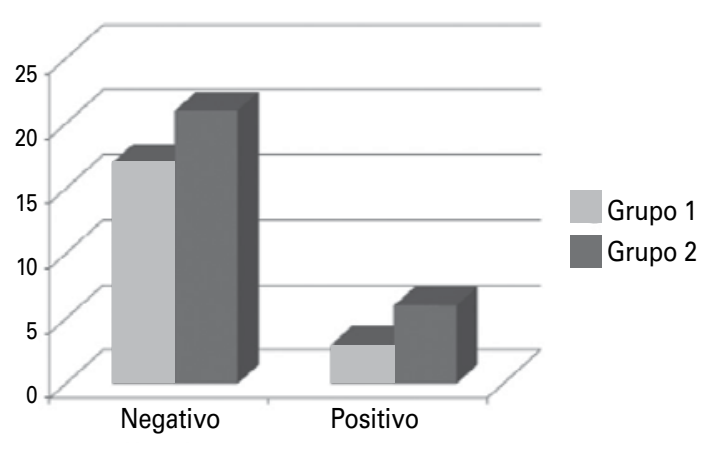

Figura 11. Distribución por Diabetes mellitas.

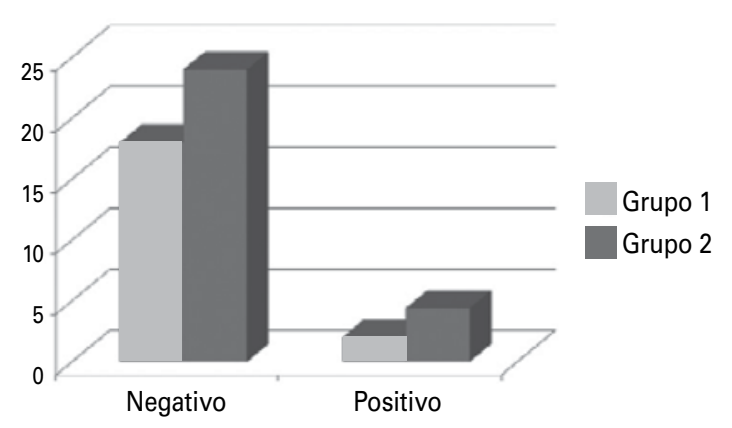

Figura 12. Distribución por Hipertensión arterial.

\section{REFERENCIAS}

1. Mixter WJ, Barr JS. Rupture of the intervertebral disc with involvement of the spinal canal. N Engl J Med. 1934;211:210-25

2. Wisneki RJ, Garfin SR, Rothman RH y Lutz GE. Discopatía lumbar. In: Herkowitz HN, Rothman RH, Simeone FA. Columna vertebral. Traducción de González JL, Sapiña S. 4a. edición. México: McGraw Hill. 2000. p. $629-31$.

3. Nachemson AL. The lumbar spine: an orthopaedic challenge. Spine. 1976;1(1):59-71.

4. Awad JN, Moskovich R. Lumbar disc herniations: surgical versus nonsurgical treatment. Clin Orthop Relat Res. 2006;(443): 183-197

5. Weinstein JN Lurie JD, Tosteson TD, Tosteson AN, Blood EA Abdu WA et al Surgical versus nonoperative treatment for lumbar disc herniations: four - year results for the Spine Patient Outcomes Research Trial (SPORT). Spine (Phila Pa 1976). 2008;33(25):2789-800.

6. Mariconda M, Galasso O, Secondulfo V, Rotonda GD, Milano C. Minimum 25-year outcome and functional assessment of lumbar discectomy. Spine (Phila Pa 1976). 2006:31(22):2593-9.

7. Lurie JD, Berven SH, Gibson-Chambers J, Tosteson T, Tosteson A, Hu SS, et al. Patient preferences and expectations for care: determinants in patients with lumbar intervertebral disc herniation. Spine (Phila Pa 1976). 2008;33(24):2663-8

8. Dewing CB, Provencher MT, Riffenburgh RH, Kerr S, Manos RE. The outcomes of lumbar microdiscectomy in a young, active population: correlation by herniation type and level. Spine (Phila Pa 1976). 2008;33(1):33-8

9. Kleinstück FS, Grob D, Lattig F, Bartanusz V, Porchet F, Jeszenszky D, et al. The influence of preoperative back pain on the outcome of lumbar decompression surgery. Spine (Phila $\mathrm{Pa}$ 1976). 2009;34(11):1198-203

10. Atlas SJ, Chang Y, Keller RB, Singer DE, Wu YA, Deyo RA. The impact of disability compensation on long-term treatment outcomes of patients with sciatica due to a lumbar disc herniation. Spine (Phila Pa 1976). 2006;31(26):3061-9.

11. Kim MS, Park KW, Hwang C, Lee YK, Koo KH, Chang BS, et al.Recurrence rate of lumbar disc herniation after open discectomy in active young men. Spine (Phila Pa 1976). 2009:34(1):24-9.

12. McGirt MJ, Ambrossi GL, Datoo G, Sciubba DM, Witham TF, Wolinsky JP, et al. Recurrent disc herniation and long-term back pain after primary lumbar discectomy: review of outcomes reported for limited versus aggressive disc removal. Neurosurgery. 2009;64(2):338-44

13. Williams RW. Microlumbar discectomy: A conservative surgical approach to the virgin herniated lumbar disc. Spine (Phila Pa 1976). 1978;3(2):175-82

14. Spengler DM. Lumbar discectomy. Results with limited disc excisión and selective foraminotomy. Spine (Phila Pa 1976). 1982;7(6):604-7.

15. O'Conell JE. Protrusions of the lumbar entervertebral disc, a clinical review based on five hundred cases treated by excision of the protrusion. J Bone Joint Surg $\mathrm{Br}$. 1951:33(1):8-30.

16. Papadopoulos EC, Girardi FP, Sandhu HS, Sama AA, Parvataneni HK, O'Leary PF, et al. Outcome of revision discectomies following recurrent lumbar disc herniation. Spine (Phila Pa 1976). 2006;31(13):1473-6.

17. Cheung KM, Karppinen J, Chan D, Ho DW, Song YQ, Sham P,et al. Prevalence and pattern of lumbar magnetic resonance imaging changes in a population study of one thousand forty-three individuals. Spine (Phila Pa 1976). 2009;34(9):934-40.

18. Lurie JD, Doman DM, Spratt KF, Tosteson AN, Weinstein JN. Magnetic resonance imaging interpretation in patients with symptomatic lumbar spine disc herniations: comparison of o el defecto del anillo fibroso, como el caso de celulosa regenerada oxidizada, que provoca fibrosis en el defecto anular y que actúa como barrera para la recurrencia de hernia discal ${ }^{35}$

El uso de implantes interespinosos aun continua siendo controversial y faltan estudios controlados prospectivos para evaluar su eficacia clínica ${ }^{36}$

\section{CONCLUSIONES}

El tratamiento quirúrgico para la hernia discal sintomática, tanto primaria como recurrente ofrece muy buenos resultados, resultados que superan a los obtenidos con el manejo conservador, aún a largo plazo. Por lo que a pesar de encontrar factores de riesgo para recurrencia, es prudente ofrecer como primera alternativa la excisión quirúrgica del disco.

Nosotros no recomendamos la fusión en caso de recurrencia, ya sea ipsi o contralateral, a menos de que esté demostrada la presencia de inestabilidad concomitante; esto debido a que se prolonga el tiempo de exposición quirúrgica y con ello aumenta la posibilidad de presentar dolor lumbar residual importante, además del aumento del costo económico.

Reviste mucha importancia la capacidad de detectar la presencia de fibrosis postquirúrgica, así como el diagnóstico oportuno de recurrencia, para lo cual nos valemos de los datos clínicos y de los recursos de imagenología disponibles. Teniendo como principal grupo de pacientes a aquellos que se encuentran en la etapa más productiva laboralmente hablando, es muy importante realizar un diagnóstico precoz y la resolución temprana del problema.

Será interesante estudiar las posibles mejorías de nuestros resultados con la aplicación de criterios diagnósticos más objetivos y de implantes tecnológicos como los dispositivos interespinosos, con la finalidad de disminuir a su expresión mínima los resultados no satisfactorios de la cirugía para hernia discal.

clinician and radiologist readings. Spine (Phila Pa 1976). 2009:34(7):701-5.

19. Lurie JD, Tosteson AN, Tosteson TD, Carragee E, Carrino JA, Kaiser JN, et al. Reliability of magnetic resonance imaging readings for lumbar disc herniation in the Spine Patient Outcomes Research Trial (SPORT). Spine (Phila Pa 1976). 2008;33(9):991-8.

20. Tampier C, Drake JD, Callaghan JP, McGill SM. Progressive disc herniation: an investigation of the mechanism using radiologic, histochemical, and microscopic dissection techniques on a porcine model. Spine (Phila Pa 1976). 2007;32(25):2869-74

21. Cinotti G, Roysam GS, Eisenstein SM, Postacchini F. Ipsilateral recurrent lumbar disc herniation. A prospective, controlled study. J Bone Joint Surg Br. 1998;80(5):825-32.

22. Loupasis GA, Stamos K, Katonis PG, Sapkas G, Korres DS, Hartofilakidis G. Seven- to 20year outcome of lumbar discectomy. Spine (Phila Pa 1976). 1999;24(22):2313-7.

23. Chang SB, Lee SH, Ahn Y, Kim JM. Risk factor for unsatisfactory outcome after lumbar foraminal and far lateral microdecompression. Spine (Phila Pa 1976). 2006;31(10):1163-7.

24. Carragee EJ, Han MY, Suen PW, Kim D. Clinical outcomes after lumbar discectomy for sciatica: the effects of fragment type and anular competence. J Bone Joint Surg Am. 2003:85(1):102-8.

25. McGirt MJ, Eustacchio S, Varga P, Vilendecic M, Trummer M, Gorensek M, et al. A prospective cohort study of close interval computed tomography and magnetic resonance imaging after primary lumbar discectomy: factors associated with recurrent disc herniation and disc height loss. Spine (Phila Pa 1976). 2009;34(19):2044-51.

26. Yorimitsu E Chiba K, ToyamaY, Hirabayashi K. Long-term outcomes of standard discectomy for lumbar disc herniation: a follow-up study of more than 10 years. Spine (Phila Pa 1976). 2001;26(6):652-7.

27. Jönsson B, Strömqvist B. Clinical characteristics of recurrent sciatica after lumbar discectomy. Spine (Phila Pa 1976). 1996;21(4):500-5

28. Gaston P Marshall RW. Survival analysis is a better estimate of recurrent disc herniation. J Bone Joint Surg Br. 2003:85(4):535-7.

29. Mysliwiec LW, Cholewicki J, Winkelpleck MD, Eis GP. MSU classification for herniated lumbar discs on MRI: toward developing objective criteria for surgical selection. Eur Spine J. 2010;19(7):1087-93

30. Ambrossi GL, McGirt MJ, Sciubba DM, Witham TF, Wolinsky JP, Gokaslan ZL, et al. Recurrent lumbar disc herniation after single-level lumbar discectomy: incidence and health care cost analysis. Neurosurgery. 2009;65(3):574-8.

31. Takeshima T, Kambara K, Miyata S, Ueda Y, Tamai S. Clinical and radiographic evaluation of disc excision for lumbar disc herniation with and without posterolateral fusion. Spine (Phila Pa 1976). 2000:25(4):450-6.

32. Cinotti G, Gumina S, Giannicola G, Postacchini F. Contralateral recurrent lumbar disc her niation. Results of discectomy compared with those in primary herniation. Spine (Phila Pa 1976). 1999;24(8):800-6

33. Fu TS, Lai PL, Tsai TT, Niu CC, Chen LH, Chen WJ. Long-term results of disc excision for recurrent lumbar disc herniation with or without posterolateral fusion. Spine (Phila Pa 1976). 2005:30(24):2830-4

34. Rönnberg K, Lind B, Zoëga B, Halldin K, Gellerstedt M, Brisby H. Patients' satisfaction with provided care/information and expectations on clinical outcome after lumbar disc herniation surgery. Spine (Phila Pa 1976). 2007;32(2):256-61.

35. Mastronardi L Puzzilli F Packing of intervertebral spaces with oxidized regenerated cellulose to prevent the recurrence of lumbar disc herniation. Neurosurgery. 2003;52(5):1106-9.

36. Kabir SM, Gupta SR, Casey AT. Lumbar interspinous spacers: a systematic review of clinical and biomechanical evidence. Spine (Phila Pa 1976). 2010;35(25):E1499-506. 